\title{
Some Fixed Point Theorems in Pseudo Multiplicative Metric Spaces with Convex Structure
}

\author{
Naveed Jamal ${ }^{*, 2}$, M Anwar Chaudhry ${ }^{1}$, M. Bakhsh Baloch ${ }^{2}$ \\ Department of Mathematics and Statistics ${ }^{1}$ \\ Institute of Southern Punjab (ISP) \\ Multan, Pakistan. \\ Department of Mathematics ${ }^{2}$ \\ Ghazi University D.G.Khan,Pakistan \\ E-mail: khanwaleed66@gmail.com
}

\section{Abstract}

In the framework of a multiplicative metric space. We have discuss some Properties of convex structures in pseudo multiplicative metric space and have applied these properties to obtain a fixed point results in complete pseudo It would be interesting to prove some further results in such metric spaces with completeness property.

Keywords: contractive mapping; Convex Pseudo multiplicative metric space; fixed point theorems

\section{Introduction and Preliminaries}

Fixed point theory is one of the most dynamic research area and has many applications in differential and integral equations. The most impressing result in this area was given by Banach in 1922. This result is called Banach contraction principle. Every contraction has a unique fixed point in a complete metric space. Due to elementary technique used in the proof by Banach and its usefulness in solving various problems in many areas, efforts have been made for generalizing this celebrated result in many abstract spaces and for various operators.

In 1906, French mathematician M. Frechet carried axiomatic development of the notion of a matric space. The metric spaces play an important role in the growth of Functional Analysis.Inspired by the impact of the notion of a metric space in Mathematics, several researchers attempted various generalizations of this notion such as rectangular metric spaces,semimetric spaces, quasi-metric spaces,

Moreover Eniola Funmilayo kazeem [9] the concept of a quasi- pseudometric space was introduced and some fixed point theorem was proven. The concept of a b-metric space has been studied Czerwick in $[4,5]$ and many others obtained many fixed point results for single and multi-value mappings.

In 1970, Takahashi [15-16] introduced convexity in metric spaces and studied certain fixed-point theorems in such metric spaces for non-expansive maps. 
The convex structure on convex spaces was studied by several authors after that. Ding[8] dealt with the iteration scheme of Ishikawa in 1988 to construct fixed points of qusi-contractive, generalized qusi-contractive and qusi -non expansive maps in convex metric spaces. In 1989, Sahab and Khan [13] extended some known results of best approximation in spaces with convex structure. In 1998, Beg [1] proved the existence of fixed points in uniformly convex complete metric spaces.

In 2003, Change and Kim et al. [2] demonstrated some convergence theorems of the iterative sequences of Ishikawas type with errors metric $s$ paces for nonlinear generalized quasi-contractive maps.In 2008,Ciric et al. [3] showed the strong convergence of Mann's iterative type process for pseudo-contractive maps in Hilbert spaces; in the same year, Wang and Liu used the iterative type process of Ishikawa with errors to approximate the fixed point of two uniformly quasi-Lipschitzain maps in convex metric spaces.

Fixed Point theorems in pseudo multiplicative metric space with convex structure are being investigated now days. The purpose of this paper to find some new fixed point theorems in these metric spaces.

Definition 1.[Pseudo Metric Space]

Let $X$ is a non- empty set. A function $D: X \times X \rightarrow \mathbb{R}^{+}$is called a Pseudo multiplicative metric. If for any $X, y, z \in X$ is satisfied with the following conditions:

$\left(N_{1}\right) \quad \mathrm{D}(\mathrm{x}, \mathrm{x})=0$,

$\left(N_{2}\right) \quad \mathrm{D}(\mathrm{x}, \mathrm{y})=\mathrm{D}(\mathrm{y}, \mathrm{x})$,

$\left(N_{3}\right) \quad \mathrm{D}(\mathrm{x}, \mathrm{z}) \leq \mathrm{D}(\mathrm{x}, \mathrm{y})+\mathrm{D}(\mathrm{y}, \mathrm{z}) . \quad$ for all $\mathrm{x}, \mathrm{y}, \mathrm{z} \in \mathrm{X}$

Definition 2.[Pseudo Multiplicative Metric Space]

Let $X$ is a set. A function $D: X \times X \rightarrow \mathbb{R}^{+}$is called a Pseudo multiplicative metric if for any $x, y, z \in X$, the following condition hold:

$\left(t_{1}\right) \quad \mathrm{D}(\mathrm{x}, \mathrm{x})=1$,

$\left(t_{2}\right) \quad \mathrm{D}(\mathrm{x}, \mathrm{y})=\mathrm{D}(\mathrm{y}, \mathrm{x})$,

$\left(t_{3}\right) \quad \mathrm{D}(\mathrm{x}, \mathrm{z}) \leq \mathrm{D}(\mathrm{x}, \mathrm{y}) \cdot \mathrm{D}(\mathrm{y}, \mathrm{z})$.

Then it is said that function $D$ is a Pseudo Multiplicative metric on $X$.

Definition 3. Let $(X, D)$ be a pseudo multiplicative metric space

Assume that $\left\{x_{n}\right\}$ is a sequence in $\mathrm{X}$ and $\mathrm{X} \in \mathrm{X}$. Then

(i) The sequence $\left\{x_{n}\right\}$ in $\mathrm{X}$ is said to be convergent $\mathrm{X}$, if

$$
\operatorname{Lim}_{n \rightarrow \infty} d(x, x)=1
$$

(ii) The sequence $\left\{x_{n}\right\}$ in $\mathrm{X}$ is said to be a Cauchy Sequence If for any

$\epsilon>1$, there exists a positive integer $n_{0}$ such that $\mathrm{d}\left(x_{n}, x_{k}\right)<\epsilon$

for all $n, k>n_{0}$

(iii) The Pseudo multiplicative metric space is said to be complete if every

Cauchy sequence converges to a point in it.

(iv) Let $T: X \rightarrow X$ be a mapping. The mapping $T$ is said to be

sequentially continuous if $x_{n} \rightarrow \mathrm{x}$ implies $\mathrm{T}\left(x_{n}\right) \rightarrow \mathrm{T}(\mathrm{x})$, as $\mathrm{n} \rightarrow \infty$.

Definition 4.[Multiplicative Contraction Mapping]

Let $(X, D)$ be a multiplicative pseudo metric space. A map $T: X \rightarrow X$ is said to be a contraction if there a exists a number $\lambda$ with $0<\lambda \leq 1$ such that $\mathrm{D}(\mathrm{Tx}, \mathrm{Ty}) \leq[D(x, y)]^{\lambda}$ 


\section{Definition 5.}

(i) A point $x \in X$, if $T x=x$, is a fixed point of $T$

(ii) It is said that a point $x \in X$ is a common fixed point of $T$ and $S$ :

If $T \mathrm{x}=\mathrm{S} \mathrm{x}=\mathrm{x}$ when $\mathrm{T}, \mathrm{S}: \mathrm{X} \rightarrow \mathrm{X}$ are mappings.

Definition 6.[Self Mapping]

Let's make $T$ a set.. A self - mapping on $T$ maps itself from $T$.

\section{Definition 7.}

Suppose $(X, D)$ is a multiplicative pseudo metric space.

A mapping $W: X \times X \times[0,1] \rightarrow X$ is said to be convex on $X$, if for each $(\mathrm{x}, \mathrm{y}, \lambda) \in \mathrm{X} \times \mathrm{x} \times[0,1]$ and $\mathrm{u} \in \mathrm{x}$

$\mathrm{D}(\mathrm{u}, \mathrm{w}(\mathrm{x}, \mathrm{y}, \lambda)) \leq D(u, x)^{\lambda} \cdot D(u, x)^{1-\lambda}$.

A Pseudo-

Multiplicative Space $X$ equipped with Convex structure $W$ is called Convex $P$ seudo-Multiplicative Space. Denoted (X,D,W).

\section{Definition 8.}

A sequence $\left\{x_{n}\right\}$ is multiplicative Cauchy, if for each $\in>1$, there exists $N_{0} \in$ $\mathrm{N}$ such that $\mathrm{D}\left(x_{n}, x_{m}\right)<\in$ for each $\mathrm{m}, \mathrm{n} \geq N_{0}$

\section{Definition 9.}

A mapping $\mathrm{T}: \mathrm{X} \rightarrow \mathrm{X}$ it is said to be continuous multiplicative at the point $\mathrm{X} \in \mathrm{X}$ iff $\mathrm{T}\left(x_{n}\right) \rightarrow \mathrm{T}(\mathrm{X})$ for every sequence $\left\{x_{n}\right\}$ with $x_{n} \rightarrow \mathrm{X}$.

\section{Definition 10.}

Let $E$ be a non- empty subset of Multiplicative Space $X$. A mapping $T: E \rightarrow E$ is said to be a k-Lipschitzian if there exists a

$\mathrm{k} \in[0, \infty)$ such that $\mathrm{D}(\mathrm{Tx}, \mathrm{Ty}) \leq \mathrm{D}(x, y) \mathrm{K}, \quad \forall \mathrm{x}, \mathrm{y} \in \mathrm{E}$.

Theorem . [11] Let $\left(X, D_{p}\right)$ be a metric space of multiplication. Define

$$
\begin{aligned}
& D_{p}: \mathrm{X} \times \mathrm{X} \rightarrow[0,+\infty] \text { by } \\
& D_{p}(\mathrm{x}, \mathrm{y})=\ln (\mathrm{p}(\mathrm{x}, \mathrm{y}))
\end{aligned}
$$

Then $\left(\mathrm{X}, D_{p}\right)$ is a metric space.

Theorem :[12] Let $(X, D)$ be a metric space of multiplication and let $f: X \rightarrow X$ be a multiple contraction. If $(X, D)$ is complete, $f$ has a fixed point that is unique.

Lemma2.1 :-Let $(X, D, W)$ be a convex Pseudo multiplicative metric space for all $x, y$ $\in \mathrm{X}, \lambda \in[0,1)$ the following property are satisfied:

a) $\quad D(x, y)=D(x, w(x, y),) \cdot D(w(x, y), y$,

b) $\quad \mathrm{D}(\mathrm{x}, \mathrm{w}(\mathrm{x}, \mathrm{y}, \lambda))=\mathrm{D}(x, y)^{1-\lambda}$

c) $\quad \mathrm{D}(\mathrm{y}, \mathrm{w}(\mathrm{x}, \mathrm{y}, \lambda))=\mathrm{D}(x, y)^{\lambda}$

Proof:-

a) Let $\mathrm{x}, \mathrm{y} \in \mathrm{X}$. Then

by definition of convex structure

$\mathrm{D}(\mathrm{x}, \mathrm{w}(\mathrm{x}, \mathrm{y}, \lambda)) \cdot \mathrm{D}(\mathrm{w}(\mathrm{x}, \mathrm{y}, \lambda), \mathrm{y}) \leq\left(\mathrm{D}(x, x)^{\lambda} \cdot \mathrm{D}(x, y)^{1-\lambda}\right)\left(\mathrm{D}(x, y)^{\lambda} \cdot \mathrm{D}(y, y)^{1-\lambda}\right)$

because $(X, D)$ is pseudo-metric space multiplicative.

Since $D(x, x)=D(y, y)=1$, so 
$\mathrm{D}(\mathrm{x}, \mathrm{w}(\mathrm{x}, \mathrm{y}),) \cdot \mathrm{D}(\mathrm{w}(\mathrm{x}, \mathrm{y}),, \mathrm{y}) \leq(1) \mathrm{D}(x, y)^{1-\lambda} \mathrm{D}(x, y)^{\lambda}(1)=\mathrm{D}(\mathrm{x}, \mathrm{y})$

$\mathrm{D}(\mathrm{x}, \mathrm{w}(\mathrm{x}, \mathrm{y}, \lambda)) . \mathrm{D}(\mathrm{w}(\mathrm{x}, \mathrm{y}, \lambda), \mathrm{y}) \leq \mathrm{D}(\mathrm{x}, \mathrm{y})$

By $t_{3}$ we have

$\mathrm{D}(\mathrm{x}, \mathrm{y}) \leq \mathrm{D}(\mathrm{x}, \mathrm{w}(\mathrm{x}, \mathrm{y}, \lambda)) . \mathrm{D}(\mathrm{w}(\mathrm{x}, \mathrm{y}, \lambda), \mathrm{y})$

From (i) and (ii), we get

$$
D(x, y)=D(x, w(x, y, \lambda)) \cdot D(w(x, y, \lambda), y)
$$

b) For every $\mathrm{x}, \mathrm{y} \in \mathrm{X}$ Further.

$\mathrm{D}(\mathrm{x}, \mathrm{w}(\mathrm{x}, \mathrm{y}, \lambda))=\mathrm{D}(x, y)^{1-\lambda}$

By definition of Convex Structure we have

This implies $\mathrm{D}(\mathrm{x}, \mathrm{w}(\mathrm{x}, \mathrm{y}, \lambda)) \leq \mathrm{D}(x, x)^{\lambda} \cdot \mathrm{D}(x, y)^{1-\lambda}$

$$
\leq(1) \quad \mathrm{D}(x, y)^{1-\lambda}
$$

$\mathrm{D}(\mathrm{x}, \mathrm{w}(\mathrm{x}, \mathrm{y}, \lambda)) \leq \mathrm{D}(x, y)^{1-\lambda}$

and using (a) we have

$\mathrm{D}(x, y)^{1-\lambda}=\mathrm{D}(\mathrm{x}, \mathrm{y}) \cdot \mathrm{D}(x, y)^{-\lambda}$

But

$$
=[\mathrm{D}(\mathrm{x}, \mathrm{w}(\mathrm{x}, \mathrm{y}, \lambda)) \cdot \mathrm{D}(\mathrm{w}(\mathrm{x}, \mathrm{y}, \lambda), \mathrm{y})] \cdot \mathrm{D}(x, y)^{-\lambda}
$$

$\mathrm{D}(\mathrm{w}(\mathrm{x}, \mathrm{y}, \lambda), \mathrm{y}) \leq \mathrm{D}(x, y)^{\lambda} D(y, y)^{1-\lambda}$ This implies

$\mathrm{D}(\mathrm{w}(\mathrm{x}, \mathrm{y}, \lambda), \mathrm{y}) \leq \mathrm{D}(x, y)^{\lambda}$

Therefore,

$\mathrm{D}(x, y)^{1-\lambda} \leq\left[\mathrm{D}(\mathrm{x}, \mathrm{w}(\mathrm{x}, \mathrm{y}, \lambda)) . \mathrm{D}(x, y)^{\lambda}\right] \mathrm{d}(x, y)^{-\lambda}$, which gives

$\mathrm{D}(x, y)^{1-\lambda} \leq \mathrm{D}(\mathrm{x}, \mathrm{w}(\mathrm{x}, \mathrm{y}, \lambda))$

From (iii) and (iv)

Hence $\mathrm{D}(\mathrm{x}, \mathrm{w}(\mathrm{x}, \mathrm{y}, \lambda))=\mathrm{D}(x, y)^{1-\lambda}$

C) By definition of convex structure

$\mathrm{D}(\mathrm{w}(\mathrm{x}, \mathrm{y}, \lambda), \mathrm{y}) \leq \mathrm{D}(x, y){ }^{\lambda} \mathrm{D}(y, y)^{1-\lambda}$

$\leq \mathrm{D}(x, y)^{\lambda}(1)$. Thus

$\mathrm{D}(\mathrm{w}(\mathrm{x}, \mathrm{y}, \lambda), \mathrm{y}) \leq \mathrm{D}(x, y)^{\lambda}$

By $t_{3}$

$$
\begin{aligned}
& \mathrm{D}(x, y)^{\lambda} \leq[\mathrm{D}(\mathrm{x}, \mathrm{w}(\mathrm{x}, \mathrm{y}, \lambda)) \cdot \mathrm{D}(\mathrm{w}(\mathrm{x}, \mathrm{y}, \lambda), \mathrm{y})]^{\lambda} \\
& \leq\left[\left(\mathrm{D}(x, x)^{\lambda} \cdot \mathrm{D}(x, y)^{1-\lambda}\right) \cdot\left(\mathrm{D}(x, y)^{\lambda} \cdot \mathrm{D}(y, y)^{1-\lambda}\right)\right]^{\lambda} \\
& \quad \leq\left[\mathrm{D}(x, y)^{1-\lambda}(1) \cdot \mathrm{D}(x, y)^{\lambda}(1)\right]^{\lambda} \\
& \mathrm{D}(x, y)^{\lambda} \leq[\mathrm{D}(x, y)]^{\lambda}=\mathrm{D}(\mathrm{w}(\mathrm{x}, \mathrm{y}, \lambda), \mathrm{y}) \\
& \mathrm{D}(x, y)^{\lambda} \leq \mathrm{D}(\mathrm{w}(\mathrm{x}, \mathrm{y}, \lambda), \mathrm{y})
\end{aligned}
$$

From (V) and (vi), we get 
$\mathrm{D}(\mathrm{w}(\mathrm{x}, \mathrm{y}, \lambda), \mathrm{y})=\mathrm{D}(x, y)^{\lambda}$

Lemma 2.2:- Let $(M, D)$ be a Pseudo multiplicative metric Space $K \subseteq M$ be closed subset of $\mathrm{M}$. Let $\mathrm{T}: \mathrm{K} \rightarrow \mathrm{K}$ be a sequentially continuous mapping then $\mathrm{D}(\mathrm{x}, \mathrm{T}(\mathrm{K}))=1$ implies $\mathrm{x} \in \mathrm{T}(\mathrm{K})$.

Proof:- Since $T$ is a self mapping and $K$ is closed subset of $M$. So, $T(K)$ is a closed subset of $K$. Due to definition of distance of a point of $(M, D)$ from a subset of $(M, D)$. We have

$\mathrm{D}(\mathrm{x}, \mathrm{T}(\mathrm{k}))=\inf \{\mathrm{D}(\mathrm{x}, \mathrm{y}): \mathrm{y} \in \mathrm{T}(\mathrm{k})\}$

and since it is given that $\mathrm{D}(\mathrm{x}, \mathrm{T}(\mathrm{k}))=1$, therefore

$D(x, T(k))=\inf \{D(x, y): y \in T(k)=1$

Sequentially continuity of $\mathrm{T}$ implies that there exists at least one distance between $x, y$ such that $D(x, y)=1$,

which in turn implies that $x$ is a limit point of $T(k)$. Since $T(k)$ is a closed subset of $M$,therefore $x \in T(k)$.

Lemma 2.3:- Let $(X, D)$ be a convex multiplicative metric space, then the following hold:

i) $\quad \mathrm{D}(\mathrm{x}, \mathrm{y})=\mathrm{D}\left(\mathrm{x}, \mathrm{w}\left(\mathrm{x}, \mathrm{y}, \frac{1}{2}\right)\right) \cdot \mathrm{D}\left(\mathrm{w}\left(\mathrm{x}, \mathrm{y}, \frac{1}{2}\right), \mathrm{y}\right)$,

ii) $\quad \mathrm{D}\left(\mathrm{x}, \mathrm{w}\left(\mathrm{x}, \mathrm{y}, \frac{1}{2}\right)\right)=\mathrm{D}\left(\mathrm{y}, \mathrm{w}\left(\mathrm{x}, \mathrm{y}, \frac{1}{2}\right)\right)=\mathrm{D}(x, y)^{\frac{1}{2}} \quad$ For all $\mathrm{x}, \mathrm{y} \in \mathrm{X}$

Proof:- (i) Let $x, y \in X$, By definition of convexness.

$$
\mathrm{D}\left(\mathrm{x}, \mathrm{w}\left(\mathrm{x}, \mathrm{y}, \frac{1}{2}\right)\right) \cdot \mathrm{D}\left(\mathrm{w}\left(\mathrm{x}, \mathrm{y}, \frac{1}{2}\right), \mathrm{y}\right) \leq\left(\mathrm{D}(x, x)^{\frac{1}{2}} \cdot \mathrm{D}(x, y)^{1-\frac{1}{2}}\right) \cdot\left(\mathrm{D}(x, y)^{\frac{1}{2}} \cdot \mathrm{D}(y, y)^{1-\frac{1}{2}}\right)
$$

Since $(X, D)$ is a multiplicative metric space,so

$$
\begin{aligned}
& \begin{aligned}
\mathrm{D}(\mathrm{x}, \mathrm{x})=\mathrm{D}(\mathrm{y}, \mathrm{y}) & =1 \\
& \left.\leq(1) \cdot \mathrm{D}(x, y)^{1-\frac{1}{2}}\right) \cdot\left(\mathrm{D}(x, y)^{\frac{1}{2}} \cdot(1) .\right. \text { Thus }
\end{aligned} \\
& \mathrm{D}\left(\mathrm{x}, \mathrm{w}\left(\mathrm{x}, \mathrm{y}, \frac{1}{2}\right)\right) \cdot \mathrm{D}\left(\mathrm{w}\left(\mathrm{x}, \mathrm{y}, \frac{1}{2}\right), \mathrm{y}\right) \leq \mathrm{D}(x, y)^{1-\frac{1}{2}} \cdot \mathrm{D}(x, y)^{\frac{1}{2}} \text {. so } \\
& \mathrm{D}\left(\mathrm{x}, \mathrm{w}\left(\mathrm{x}, \mathrm{y}, \frac{1}{2}\right)\right) \cdot \mathrm{D}\left(\mathrm{w}\left(\mathrm{x}, \mathrm{y}, \frac{1}{2}\right), \mathrm{y}\right) \leq \mathrm{D}(\mathrm{x}, \mathrm{y}) \\
& \text { By definition of Convexness }
\end{aligned}
$$

From (i) and (ii) we get

$$
D(x, y) \leq D\left(x, w\left(x, y, \frac{1}{2}\right)\right) . D\left(w\left(x, y, \frac{1}{2}\right), y\right)
$$

$$
D(x, y)=D\left(x, w\left(x, y, \frac{1}{2}\right)\right) \cdot D\left(w\left(x, y, \frac{1}{2}\right), y \quad \text { for all } x, y \in X\right.
$$

(ii) Let $x, y \in X$, By definition of convexness.

$$
\begin{array}{r}
\mathrm{D}\left(\mathrm{x}, \mathrm{w}\left(\mathrm{x}, \mathrm{y}, \frac{1}{2}\right)\right) \leq \mathrm{D}(x, x)^{1-\frac{1}{2}} \cdot \mathrm{D}(x, y)^{\frac{1}{2}} \\
\mathrm{D}\left(\mathrm{x}, \mathrm{w}\left(\mathrm{x}, \mathrm{y}, \frac{1}{2}\right)\right) \leq \mathrm{D}(x, y)^{\frac{1}{2}}
\end{array}
$$

and on the other hand

$$
\begin{aligned}
& \mathrm{D}(x, y)^{1-\frac{1}{2}}=\mathrm{D}(\mathrm{x}, \mathrm{y}) \cdot \mathrm{D}(x, y)^{-\frac{1}{2}} \\
& \mathrm{By}(\mathrm{i}) \text { we have } \\
& \mathrm{D}(x, y)^{\frac{1}{2}}=\left[\mathrm{D}\left(\mathrm{x}, \mathrm{w}\left(\mathrm{x}, \mathrm{y}, \frac{1}{2}\right)\right) \cdot \mathrm{D}\left(\mathrm{y}, \mathrm{w}\left(\mathrm{x}, \mathrm{y}, \frac{1}{2}\right)\right] \cdot \mathrm{D}(x, y)^{-\frac{1}{2}}\right.
\end{aligned}
$$

From $(\mathrm{A}), \mathrm{D}\left(\mathrm{y}, \mathrm{w}\left(\mathrm{x}, \mathrm{y}, \frac{1}{2}\right) \leq \mathrm{D}(x, y)^{\frac{1}{2}}\right.$

$$
\begin{aligned}
& \leq\left[\mathrm{D}\left(\mathrm{x}, \mathrm{w}\left(\mathrm{x}, \mathrm{y}, \frac{1}{2}\right)\right) \cdot \mathrm{D}(x, y)^{\frac{1}{2}}\right] \cdot \mathrm{D}(x, y)^{-\frac{1}{2}} \\
\mathrm{D}(x, y)^{\frac{1}{2}} & \leq \mathrm{D}\left(\mathrm{x}, \mathrm{w}\left(\mathrm{x}, \mathrm{y}, \frac{1}{2}\right)\right)
\end{aligned}
$$

From $(\mathrm{A})$ and $(\mathrm{B})$ we get 


$$
\begin{array}{ll} 
& \mathrm{D}\left(\mathrm{x}, \mathrm{w}\left(\mathrm{x}, \mathrm{y}, \frac{1}{2}\right)\right)=\mathrm{D}(x, y)^{\frac{1}{2}} \\
\text { Similarly, } & \mathrm{D}\left(\mathrm{y}, \mathrm{w}\left(\mathrm{x}, \mathrm{y}, \frac{1}{2}\right)\right)=\mathrm{D}(x, y)^{\frac{1}{2}} \\
\text { Hence } & \mathrm{D}\left(\mathrm{x}, \mathrm{w}\left(\mathrm{x}, \mathrm{y}, \frac{1}{2}\right)\right)=\mathrm{D}\left(\mathrm{y}, \mathrm{W}\left(\mathrm{x}, \mathrm{y}, \frac{1}{2}\right)\right)=\mathrm{D}(x, y)^{\frac{1}{2}}
\end{array}
$$

\section{Main Results}

In this section we discuss some fixed point theorems in Pseudo Multiplicative metric spaces with convex structure.

Theorem 1:- Let $Y$ be a non-empty closed convex subset of a complete Convex Pseudo multiplicative metric space $(X, D, W)$ and $f$ be a sequentially continuous self mapping of $Y$. if there exists

$\mathrm{k} \in\left[0, \frac{1}{2}\right)$ such that

$$
\mathrm{D}(\mathrm{x}, \mathrm{f}(\mathrm{y})) . \mathrm{D}(\mathrm{f}(\mathrm{x}), \mathrm{f}(\mathrm{y})) \leq \mathrm{D}(y, f(x))^{k}
$$

for all $x, y \in Y$.Then $f$ has at least one point fixed.

Proof :-Using Pseudo multiplicative convex structure definition we have

$$
\mathrm{D}(\mathrm{x}, \mathrm{W}(\mathrm{x}, \mathrm{y}, \lambda)) \leq \mathrm{D}(x, x)^{\lambda} . \mathrm{D}(x, y)^{1-\lambda}
$$
and

$$
\mathrm{D}(\mathrm{y}, \mathrm{W}(\mathrm{x}, \mathrm{y},)) \leq \mathrm{D}(y, x)^{\lambda} \cdot \mathrm{D}(y, y)^{1-\lambda}
$$

Since $D(x, x)=D(y, y)=1$ Consequently (2) and (3)

$$
\mathrm{D}(\mathrm{x}, \mathrm{W}(\mathrm{x}, \mathrm{y}, \lambda)) \cdot \mathrm{D}(\mathrm{y}, \mathrm{w}(\mathrm{x}, \mathrm{y}, \lambda)) \leq \mathrm{D}(\mathrm{x}, \mathrm{y})
$$

Now let $x_{0} \in \mathrm{Y}$. Define $\left\{x_{n}\right\}$ sequence as follows.

$$
x_{n}=\mathrm{w}\left(x_{n-1}, \mathrm{f} x_{n-1}, \lambda\right) \quad ; \mathrm{n}=1,2,3, \ldots \ldots \ldots
$$

Since $Y$ is convex. so $x_{n} \in Y$ and using (2), (4) and (5), we have

$$
\begin{aligned}
& \mathrm{D}\left(x_{n}, x_{n+1}\right) \leq \mathrm{D}\left(x_{n}, x_{n}\right)^{\lambda} . \mathrm{D}\left(x_{n}, f x_{n}\right)^{1-\lambda} \text { and } \\
& \mathrm{D}\left(f x_{n}, f x_{n+1}\right) \leq \mathrm{D}\left(x_{n}, f x_{n}\right)^{\lambda} \cdot \mathrm{D}\left(f x_{n}, f x_{n}\right)^{1-\lambda}
\end{aligned}
$$

Multiplying (6) and (7)

$$
\mathrm{D}\left(x_{n}, x_{n+1}\right) \cdot \mathrm{D}\left(\mathrm{f} x_{n}, x_{n+1}\right) \leq \mathrm{D}\left(x_{n}, f x_{n}\right)
$$

Now using $t_{3}$, we obtain

$$
\mathrm{D}\left(x_{n}, f x_{n}\right) \leq \mathrm{D}\left(x_{n}, f x_{n-1}\right) \cdot \mathrm{D}\left(\mathrm{f} x_{n-1}, f x_{n}\right)
$$

From (8) and (9),we get

$$
\mathrm{D}\left(x_{n}, x_{n+1}\right) \cdot \mathrm{D}\left(f x_{n}, x_{n+1}\right) \leq \mathrm{D}\left(x_{n}, f x_{n-1}\right) \cdot \mathrm{D}\left(f x_{n-1}, f x_{n}\right)
$$

This implies

$$
\frac{\mathrm{D}\left(x_{n}, x_{n+1}\right) \cdot \mathrm{D}\left(f x_{n}, x_{n+1}\right)}{\mathrm{D}\left(x_{n}, f x_{n-1}\right)} \leq \mathrm{D}\left(f x_{n-1}, f x_{n}\right)
$$

By substituting $\mathrm{x}$ for $x_{n}$ and $\mathrm{y}$ for $x_{n-1}$ in (1),we have 
This implies that

$$
\mathrm{D}\left(x_{n}, x_{n+1}\right) \cdot \mathrm{D}\left(f x_{n}, x_{n+1}\right) \leq \mathrm{D}\left(x_{n}, f x_{n-1}\right)
$$

$$
\mathrm{D}\left(f x_{n}, f x_{n-1}\right) \leq \frac{D\left(x_{n-1, x_{n}}\right)^{, k}}{D\left(f x_{n}, f x_{n-1}\right)}
$$

Using (10) and (11)

$$
\mathrm{D}\left(x_{n}, x_{n+1}\right) \cdot \mathrm{D}\left(x_{n+1}, f x_{n}\right) \leq D\left(x_{n-1}, f x_{n}\right)^{k}
$$

Again using $t_{3}$, we get

$$
\mathrm{D}\left(x_{n-1}, f x_{n}\right) \leq \mathrm{D}\left(x_{n-1}, x_{n}\right) \cdot \mathrm{D}\left(x_{n}, f x_{n}\right)
$$

Combining (12) and (13), we have

By $t_{3}$, we have

$$
\mathrm{D}\left(x_{n}, x_{n+1}\right) \cdot \mathrm{D}\left(x_{n+1}, f x_{n}\right) \leq\left[D\left(x_{n-1}, x_{n}\right) \cdot \mathrm{D}\left(x_{n}, f x_{n}\right)\right]^{k}
$$

$$
\begin{aligned}
& \mathrm{D}\left(x_{n}, f x_{n}\right) \leq D\left(x_{n-1}, x_{n}\right)^{k} . D\left(x_{n}, f x_{n}\right)^{k} \\
& D\left(x_{n}, f x_{n}\right)^{1-k} \leq D\left(x_{n-1}, x_{n}\right)^{k}
\end{aligned}
$$

Using pseudo multiplicative convex structure, we have

That is,

$$
\mathrm{D}\left(x_{n}, x_{n+1}\right) \leq D\left(x_{n}, f x_{n}\right)^{1-\lambda} \leq \mathrm{D}\left(f x_{n}, x_{n}\right)
$$

$$
\mathrm{D}\left(x_{n}, x_{n+1}\right) \leq \mathrm{D}\left(x_{n}, f x_{n}\right)
$$

Thus

$$
\begin{aligned}
& D\left(x_{n}, x_{n+1}\right)^{1-k} \leq D\left(x_{n-1}, x_{n}\right)^{k} \\
& \mathrm{D}\left(x_{n}, x_{n+1}\right) \leq D\left(x_{n-1}, x_{n}\right)^{\frac{k}{1-k}} \text {, which implies } \\
& \mathrm{D}\left(x_{n}, x_{n+1}\right) \leq D\left(x_{n-1}, x_{n}\right)^{\theta} ; \quad \text { where } \theta=\frac{k}{1-k}
\end{aligned}
$$

so,

$$
\mathrm{D}\left(x_{n}, x_{n+1}\right) \leq D\left(x_{0}, x_{1}\right)^{\theta^{n}}
$$

Similarly, we have

$$
\mathrm{D}\left(x_{n+1}, x_{n+2}\right) \leq D\left(x_{0}, x_{1}\right) \theta^{n+1}
$$

Continuing in similar way we obtain

$\mathrm{D}\left(x_{n+k-1}, x_{n+k}\right) \leq D\left(x_{0}, x_{1}\right)^{\theta^{n+k-1}}$

Using $t_{3}$, we obtain

$$
\begin{aligned}
\mathrm{D}\left(x_{n}, x_{n+k}\right) \leq & \mathrm{D}\left(x_{n}, x_{n+1}\right) \cdot \mathrm{D}\left(x_{n+1}, x_{n+2}\right) \ldots \ldots \ldots . \mathrm{D}\left(x_{n+k-1}, x_{n+k}\right) \\
& \leq D\left(x_{0}, x_{1}\right)^{\left(\theta^{n}+\theta^{n+1}+\theta^{n+2}+\ldots \ldots+\theta^{n+k-1}\right)} \\
& \leq D\left(x_{0}, x_{1}\right)^{\frac{\theta^{n}}{\theta^{n}}}
\end{aligned}
$$

Since $0 \leq \theta<1$ therefore we get $\mathrm{D}\left(x_{0}, x_{1}\right)=1$ as $\mathrm{n} \rightarrow \infty$ 


$$
\lim _{n \rightarrow \infty} d\left(x_{n}, x_{n+k}\right)=1
$$

This implies that $\left\{x_{n}\right\}$ is a Sequence Cauchy in $\mathrm{Y}$. Since $\mathrm{Y}$ is closed subset of complete space (X,D).so $Y$ is also complete subspace.

Consequently $\left\{x_{n}\right\}_{n=1}^{\infty}$ Converges to a point $\mathrm{z}$ in $\mathrm{Y}$.

$$
\lim _{n \rightarrow \infty} x_{n}=z
$$

Now we prove that $z$ is a fixed point of $f$. Because $f$ is continuous sequentially multiplicative this implies

$$
x_{n} \rightarrow \mathrm{z} \Rightarrow \mathrm{f} x_{n} \rightarrow \mathrm{fz} \text { as } \mathrm{n} \rightarrow \infty
$$

By $t_{3}$, we have

$\mathrm{D}\left(x_{n}, \mathrm{f} x_{n}\right) \leq D\left(f x_{n-1}, f x_{n-1}\right)^{\lambda} . D\left(x_{n-1,} f x_{n-1}\right)^{1-\lambda}$,

Which implies

$\mathrm{D}\left(x_{n}, \mathrm{f} x_{n}\right) \leq D\left(x_{n-1,} f x_{n-1}\right)^{1-\lambda}$

Letting $\mathrm{n} \rightarrow \infty$ We're getting

$\mathrm{D}(\mathrm{z}, \mathrm{fz}) \leq D(z, T z)^{1-\lambda} \leq \mathrm{D}(\mathrm{z}, \mathrm{fz})$

Hence $D(z, f z)=1$ and we can deduce this from Lemma $2.2 \quad z=f(z)$.

Which completes the proof.

Theorem 2:- Let $Y$ be a non-empty closed convex subset of a complete Convex Pseudo multiplicative metric space $(X, D, W)$ and $f$ be a sequentially continuous self mapping of $Y$. if there exists $k \in[2,4)$ such that

$\mathrm{D}(\mathrm{x}, \mathrm{fx}) \cdot \mathrm{D}(\mathrm{y}, \mathrm{fy}) \leq \mathrm{D}(x, y)^{k}$

for all $x, y \in Y$. Then $f$ has at least one fixed point.

Proof :-Let $x_{0} \in \mathrm{Y}$. Define a sequence $\left\{x_{n}\right\}$ by

$$
x_{n}=\mathrm{w}\left(x_{n-1}, \mathrm{f} x_{n-1}, \lambda\right) \quad ; \mathrm{n}=1,2,3, \ldots \ldots \ldots .
$$

As $Y$ is convex, so $x_{n} \in Y$ and from the Lemma 2.1, We get the following equation for $\lambda=\frac{1}{2}$

and

$$
\mathrm{D}\left(x_{n}, x_{n+1}\right)=\mathrm{D}\left(x_{n}, f x_{n}\right)^{\frac{1}{2}}
$$

$$
\mathrm{D}\left(x_{n}, x_{n-1}\right)=\mathrm{D}\left(x_{n-1}, f x_{n-1}\right)^{\frac{1}{2}}
$$

By replacing $\mathrm{x}$ with $x_{n}$ and $\mathrm{y}$ with $x_{n-1}$ in equation (14) we get

$$
\begin{aligned}
& \mathrm{D}\left(x_{n}, \mathrm{f} x_{n}\right) . \mathrm{D}\left(x_{n-1}, \mathrm{f} x_{n-1}\right) \leq \mathrm{D}\left(x_{n}, x_{n-1}\right)^{k} \\
& \text { Using equations (15),(16) and (17), we have }
\end{aligned}
$$$$
\mathrm{D}\left(x_{n}, x_{n+1}\right)^{2} . \mathrm{D}\left(x_{n}, x_{n-1}\right)^{2} \leq \mathrm{D}\left(x_{n}, x_{n-1}\right)^{k}
$$

$$
\begin{aligned}
& \mathrm{D}\left(x_{n}, x_{n+1}\right)^{2} \leq \mathrm{D}\left(x_{n}, x_{n-1}\right)^{k-2} \\
& \mathrm{D}\left(x_{n}, x_{n+1}\right) \leq \mathrm{D}\left(x_{n}, x_{n-1}\right)^{\frac{k-2}{2}} \\
& \mathrm{D}\left(x_{n}, x_{n+1}\right) \leq \mathrm{D}\left(x_{n}, x_{n-1}\right)^{\lambda} \text { where } \lambda=\frac{k-2}{2}, \text { so } \\
& \mathrm{D}\left(x_{n}, x_{n+1}\right) \leq \mathrm{D}\left(x_{0}, x_{1}\right) \lambda^{n}
\end{aligned}
$$


Similarly,

$$
\mathrm{D}\left(x_{n+1}, x_{n+2}\right) \leq \mathrm{D}\left(x_{0}, x_{1}\right)^{\lambda^{n+1}}
$$

Continuing in a similar way we get

$$
\mathrm{D}\left(x_{n+k-1}, x_{n+k}\right) \leq \mathrm{D}\left(x_{0}, x_{1}\right)^{\lambda^{n+k-1}}
$$

Using $t_{3}$, we have

$$
\begin{aligned}
& \mathrm{D}\left(x_{n}, x_{n+k}\right) \leq \mathrm{D}\left(x_{n}, x_{n+1}\right) \cdot \mathrm{D}\left(x_{n+1}, x_{n+2}\right) \ldots \ldots \ldots . . \mathrm{D}\left(x_{n+k-1}, x_{n+k}\right) \\
& \leq \mathrm{D}\left(x_{0}, x_{1}\right)^{\left(\lambda^{n}+\lambda^{n+1}+\cdots+\lambda^{n+k-1}\right)} \quad \text { Thus } \\
& \mathrm{D}\left(x_{n}, x_{n+k}\right) \leq \mathrm{D}\left(x_{0}, x_{1}\right)^{\frac{\lambda^{n}}{1-\lambda}}
\end{aligned}
$$

Since $0 \leq \lambda<1$, therefore we get

$$
\lim _{n \rightarrow \infty} D\left(x_{n}, x_{n+k}\right)=1
$$

This implies that $\left\{x_{n}\right\}$ is a Cauchy Sequence in $Y$. Since $Y$ is a complete space subset closed $(\mathrm{X}, \mathrm{D})$. so $\mathrm{Y}$ is also a complete subspace consequently $\left\{x_{n}\right\}_{n=1}^{\infty}$ Converges to a point $\mathrm{Z}$ in $\mathrm{Y}$ and

$$
\lim _{n \rightarrow \infty} x_{n}=z
$$

Now we prove that $z$ is a fixed point of $f$. Because $f$ is sequentially

continuous multiplicative this implies

$$
x_{n} \rightarrow \mathrm{z} \Rightarrow \mathrm{f} x_{n} \rightarrow \mathrm{fz} \text { as } \mathrm{n} \rightarrow \infty
$$

Also due to $t_{3}$

$$
\mathrm{D}\left(x_{n}, \mathrm{f} x_{n}\right) \leq D\left(f x_{n-1}, f x_{n-1}\right)^{\lambda} . D\left(x_{n-1,} f x_{n-1}\right)^{1-\lambda}, \text { which implies }
$$

$\mathrm{D}\left(x_{n}, \mathrm{f} x_{n}\right) \leq D\left(x_{n-1}, f x_{n-1}\right)^{1-\lambda}$

Letting $\mathrm{n} \rightarrow \infty$ we have

$\mathrm{D}(\mathrm{z}, \mathrm{fz}) \leq D(z, T z)^{1-\lambda} \leq \mathrm{D}(\mathrm{z}, \mathrm{fz})$

Therefore $D(z, f z)=1$ and Lemma 2.2, $Z=f(z)$ can be deduced. Which complete the proof.

Theorem 3:-Let $Y$ be a non-empty closed convex subset of a complete Convex Pseudo multiplicative metric space $(X, D, W)$ and $f$ be a selfmapping of $Y$. If there is $a, b, c, k$ that exists

$$
\begin{aligned}
& \frac{2 b}{c} \leq \mathrm{b} \leq \frac{2(a+b+c)}{c} \\
& \mathrm{D}(x, f(x))^{a} \cdot \mathrm{D}(y, f(y))^{b} \cdot \mathrm{D}(f(x), f(y))^{c} \leq \mathrm{D}(x, y)^{k}
\end{aligned}
$$

for all $\mathrm{x}, \mathrm{y} \in \mathrm{Y}$. Then $\mathrm{f}$ has at least one point fixed.

Proof :- Let $x_{0} \in \mathrm{Y}$ is arbitrary. A sequence is defined $\left\{x_{n}\right\}_{n=1}^{\infty} \mathrm{T}$ a follows:

$$
x_{n}=\mathrm{W}\left(x_{n-1}, \mathrm{f} x_{n-1}, \frac{1}{2}\right) \quad ; \mathrm{n}=1,2,3,
$$

Since $\mathrm{Y}$ is convex, so $x_{n} \in \mathrm{Y}$ for all $\mathrm{n} \in \mathbb{N}$. By Lemma 2.1 and(19), we have

and

$$
\mathrm{D}\left(x_{n}, \mathrm{f}\left(x_{n}\right)\right)=\mathrm{D}\left(x_{n}, x_{n+1}\right)^{2}
$$




$$
\mathrm{D}\left(x_{n}, f\left(x_{n-1}\right)\right)=\mathrm{D}\left(x_{n}, x_{n-1}\right)
$$

For all $\mathrm{n} \in \mathbb{N}$, Now, by replacement $\mathrm{x}$ with $x_{n}$ and $\mathrm{y}$ with $x_{n-1}$ in (18) we get

$$
\mathrm{D}\left(x_{n}, f\left(x_{n}\right)\right)^{a} \cdot \mathrm{D}\left(x_{n-1}, f\left(x_{n-1}\right)\right)^{b} \cdot \mathrm{D}\left(f\left(x_{n}\right), f\left(x_{n-1}\right)\right)^{c} \leq \mathrm{D}\left(x_{n}, x_{n-1}\right)^{k}
$$

For all $\mathrm{n} \in \mathbb{N}$. Therefore, from (20) and (21) it follow that

$\mathrm{D}\left(x_{n}, x_{n+1}\right)^{2 a} . \mathrm{D}\left(x_{n}, x_{n-1}\right)^{2 b} . \mathrm{D}\left(f\left(x_{n}\right), f\left(x_{n-1}\right)\right)^{c} \leq \mathrm{D}\left(x_{n}, x_{n-1}\right)^{k}$

for all $\mathrm{n} \in \mathbb{N}$. Let $\mathrm{Y}$ be non-negative number. Using the inequality Triangle,(20) and (21), We have

$$
\frac{D\left(x_{n}, x_{n+1}\right)^{2 c}}{D\left(x_{n}, x_{n-1}\right)^{c}} \leq D\left(f\left(x_{n}\right), f\left(x_{n-1}\right)\right)^{c}
$$

For all $\mathrm{n} \in \mathbb{N}$. Likewise, for the case $\mathrm{Y}<0$ we have

$\mathrm{D}\left(x_{n}, x_{n+1}\right)^{2 c} . \mathrm{D}\left(x_{n}, x_{n-1}\right)^{c} \leq \mathrm{D}\left(f\left(x_{n}\right), f\left(x_{n-1}\right)\right)^{c}$

for all $\mathrm{n} \in \mathbb{N}$. That is why we have for each case

$$
\frac{D\left(x_{n}, x_{n+1}\right)^{2 c}}{D\left(x_{n}, x_{n-1}\right)^{c}} \leq D\left(f\left(x_{n}\right), f\left(x_{n-1}\right)\right)^{c}
$$

For each $\mathrm{n} \in \mathbb{N}$. Now form (22) and (23) it follows that

$\frac{D\left(x_{n}, x_{n+1}\right)^{2 a} \cdot D\left(x_{n}, x_{n-1}\right)^{2 b} \cdot D\left(x_{n}, x_{n+1}\right)^{2 c}}{D\left(x_{n}, x_{n-1}\right)^{c}} \leq D\left(x_{n}, x_{n-1}\right)^{k}$

For all $\mathrm{n} \in \mathbb{N}$. That implies

$\mathrm{D}\left(x_{n}, x_{n+1}\right) \leq \mathrm{D}\left(x_{n}, x_{n-1}\right)^{\frac{k-2 b+|c|}{2(a+c)}}$

for all $\mathrm{n} \in \mathbb{N}$. From (a) $\frac{k-2 b+|c|}{2(a+c)} \in[0,1)$ and therefore, $\left\{x_{n}\right\}_{n=1}^{\infty}$ is construction Sequence in Y.So it's Cauchy Sequence.Since $Y$ is a complete space.

there exist $\mathrm{v} \in \mathrm{Y}$ such that $\lim _{n \rightarrow \infty} x_{n}=\mathrm{v}$. Now, by applying $\mathrm{x}$ to $\mathrm{v}$ and $\mathrm{y}$ with $x_{n}$ in (18). We get

$\mathrm{D}(v, f(v))^{a} \cdot \mathrm{D}\left(x_{n}, f\left(x_{n}\right)\right)^{b} \cdot \mathrm{D}\left(f(v), f\left(x_{n}\right)\right)^{c} \leq \mathrm{D}\left(v, x_{n}\right)^{k}$

for all $\mathrm{n} \in \mathbb{N}$. Letting $\mathrm{n} \rightarrow \infty$ It follow from the above inequality

$\mathrm{D}(v, f(v))^{(a+c)} \leq 0$

Since $(\mathrm{a}+\mathrm{c})$ is positive. it follows that $\mathrm{D}(v, f(v))=1$. Hence $\mathrm{f}(\mathrm{v})=\mathrm{v}$ and complete the theorem.

\section{REFERENCES}

[1] I.Beg, Structure of the set of fixed points of non-expansive mappings on convex metric spaces, Univ. Mariae curie- Sklodowska Lublin- Polonia 2 (1998), 714.

[2] S. Chang, and J. K. Kim, Convergence theorems of the Ishikawa type iterative sequences with error for generalized Qusi-contractive mappings in convex metric spaces, App. Math. Lett. 16 (2003), 535-542. 
[3] L. Ciric, A. Rafiq, N. Cakle and J. S. Ume, Implicit Mann fixed point iterations for pseudo-contractive mappings, Accepted Manuscript in Appl. Math. Lett. (June 2008).

[4] S. Czerwick, Contraction Mapping in b-metric Spaces, Acta Math. Inform Univostrav, (1993), no. 1, 5-11.

[5] S. Czerwick, Nonlinear set valued Contraction mappings in b-metric spaces, AttiSemmin. Mat. Fis. Univ. Modern, 46 (1998), 263-276.

[6] B. K. Dass and S. Gupta, An extension of Banach contraction principle through rational expression, Indian Journal of Pure and Applied Mathematics 6 (1975), no. 12,1455-1458.

[7] B. C. Dhage, Generalized metric spaces with fixed points, Bull. Calcutta Math. Soc. 84 (1992), 329-336.

[8] X. P. Ding, Iteration processes of nonlinear mappings in convex metric spaces, J. Math. Anal, App. 132 ( 1988), 114-122.

[9] Eniola Funmilayo Kazeem "On Quasi-Pseudo metric type spaces" Hindawi Publishing corporation, volume 2014, Article ID 198685,7 pages.

[10] H. P. A. Kunzi, note on sequentially compact quasi-pseudo metric spaces, Monatsh. Math. 95 (1983), 219-220.

[11] Lazaiz al. Fixed point Theory and Applications (2017) 2017;3

[12] S. A. Mohiuddin, M. Cancan and H. Selvi, Intuitionistic fuzzy stability of a Jensen functional equation via fixed point technique, Math. Comput. Model 54 (2011), 2403- 2409.

[13] S. A. Sahab and M. S. Khan, Best approximation in spaces with convex structure, Bulletin of the institute of mathematics Academia Sinica 17 (1989). 75-79

[14] T. Suzuki, Subrahmanyams fixed point theorem, Nonlinear Analysis, 71 (2009), 1678-1683.

[15] W. Takashashi, Nonlinear functional Analysis. Fixed point theory and its applications, Yokohama Publisher 2000.

[16] W. Takahashi, A convexity in metric space and non-expansive mappings, Kodai Math, Sem. Rep. 22(1970), 142-149.

[17] J. S. Ume, Fixed point Theorem in generalizing spaces of quasi metric family and applications, international journal of Pure and Applied Mathematics, 33 (2002), 1041-1051. 